\title{
LE DÉVELOPPEMENT, L'ÉVOLUTION ETT L'AVENIR DE LA PISCICULTURE AU CAMEROUN
}

\author{
par J. BARD \\ Inspecteur des Eaux et Forêts de la France d'Outre Mer \\ (Cameroun).
}

Il n'est pas besoin d'exposer dans le cadre de cet article les buts du développement des industries piscicoles dans le Territoire du Cameroun; les diététistes ont suffisamment exposé la "faim de protéines" dont souffre la population des régions forestières d'Afrique. Cette faim les pousse même, si l'on en croit Monod (L'Industrie des pêches au Cameroun), à consommer les têtards et des insectes aquatiques. Ce qui était vrai en 1925 l'est encore aujourd'hui bien que, du fait du développement économique, le niveau de vie de la population ait sensiblement monté. En effet, l'amélioration de l'alimentation touche surtout les centres commerciaux où l'autochtone peut se procurer la viande, la boîte de lait ou de sardine, le stock-fish ou le hareng importé dont il agrémentera ses repas. Mais, hors de ces centres, la femme qui préparera la nourriture familiale ne trouvera que des tubercules avec l'assaisonnement traditionnel. Les chevreaux, cabris, moutons et poulets de l'élevage domestique ne sont consommés qu'exceptionnellement. Quant à la pêche, pratiquée quelques jours par an aux basses eaux des ruisseaux, elle ne rapporte guère que du menu fretin dans ces eaux dévastées depuis longtemps par l'usage des barrages et du poison. Les grands fleuves (Wouri, Sanaga, Nyong, Ntem, Dja) seraient sans doute poissonneux, mais leurs rives sont peu fréquentées, probablement en raison de l'insalubrité qui y règne, due à la présence des Glossines. Il n'y a pratiquement qu'une seule zone de pèche intensive au Cameroun, mais elle est bien en dehors de la région forestière puisqu'elle se situe immédiatement au sud du lac Tchad.

Si la production piscicole de cette région est très forte (on a avancé le chiffre de 40.000 tonnes de poisson frais pêché chaque année), elle n'a qu'une incidence restreinte sur l'économie du reste du Territoire, hormis les pays immédiatement avoisinants, en raison $\mathrm{du}$ prix prohibitif des transports terrestres, ainsi d'ailleurs que des cours élevés pratiqués localement, du fait de l'existence d'un commerce important avec la Nigéria voisine.

De ce qui précède, on peut déduire que l'amélioration de la pêche continentale au Cameroun est un problème assez ardu, qu'il était donc 
logique d'inaugurer l'effort piscicole par l'introduction de la pisciculture qui permet, en principe, à l'autochtone de se procurer du poisson en toute saison, à proximité de sa case, avec une technique de récolte simple.

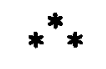

Si l'on met de côté les régions littorales (Douala, Edéa, Kribi) où la pisciculture n'a pas d'intérêt au regard de la pêche en mer et dans les estuaires, la géographie du Cameroun se présente ainsi, vu sous l'aspect " pisciculture ":

I. Les plateaux du Centre (Yaoundé, Ebolowa, Sangmélima) où le régime régulier des eaux, la relative densité de la population et l'activité économique (culture du cacaoyer) sont autant de facteurs favorables à la pisciculture, mais où la construction des étangs est rendue délicate par la configuration du relief (vallée en $\mathrm{V}$ très profondes);

II. Les plateaux de l'Est (Abong-Mbang, Batouri, Yokadouma) analogues aux précédents pour le régime des eaux et le relief, mais où la population est clairsemée;

III. Les régions montagneuses de l'Ouest (Bafia, Nkongsamba, Dschang, Foumban), où le régime régulier des eaux, la configuration douce du relief et la densité de la population, concourent au développement exceptionnel de la pisciculture;

IV. Les plateaux de l'Adamaoua (Ngaoundéré, Banyo, Tibati, Tignère, Meiganga), dont le relief, qui rappelle celui de l'Ouest, favorise la construction des étangs, mais où le régime des eaux est torrentiel, surtout vers le Nord (crues violentes en saison des pluies, asséchement des cours d'eau en saison sèche). D'autre part, comme dans l'Est, la rareté de la population et sa dispersion sont des facteurs défavorables.

Dans les autres régions, la pisciculture n'est que fort peu pratiquée, les conditions locales ne s'y prêtant que mal ou pas du tout.

Il peut paraître surprenant que, dans cette classification, on n'ait pas fait allusion à la valeur piscicole des eaux appréciée d'après leurs propriétés chimiques. Mais on peut avancer, sans erreur grossière, que cette valeur piscicole est pratiquement constante sur toute l'étendue du Territoire à une exception près. Les eaux sont uniformément pauvres : $p \mathrm{H}$ autour de 6, degré hydrotimétrique 1,5 à 3 , SBV : 0,3 à 0,6 , ce qui corrobore, d'ailleurs, la théorie de la zonalité des sols. L'exception vise les eaux des terrains volcaniques récents (pleistocène) de l'Ouest : $p \mathrm{H}$ de 7 à 8 , degré hydrotimétrique 11 à $14, \mathrm{SBV}$ autour de 2,5.

Les teneurs en oxygène dissous n'ont jusqu'à présent pu être notées, mais il ne semble pas, d'après les résultats obtenus à Yaoundé, qu'au moins pour la pisciculture du Tilapia cette teneur ait une grande influence sur le rendement des étangs. Tout au plus limite-t-elle la possibilité de 
faire de la pisciculture dans certaines eaux où les fermentations sont particulièrement actives. Les analyses protistologiques donneraient sans doute une classification plus intéressante : elles sont inscrites au programme de la Station de Yaoundé.

Jusqu'à présent, la seule pisciculture pratiquée au Cameroun est celle des Tilapia importée à la suite d'une mission, au Congo Belge, de M. Letouzey, Chef de la Section de Recherches Forestières à la fin de 1948.

Des alevins de Tilapia nigra et melanopleura furent ramenés par la route dans un fût à essence ouvert, à travers le Congo Belge, l'Oubangui et le Cameroun. La pisciculture du Tilapia nigra a été abandonnée, mais tous les Tilapia melanopleura élevés en étang au Cameroun descendent de ceux qui ont été apportés par cette voie. C'est seulement en 1950 que des alevins de Tilapia macrochir furent importés du Katanga au Cameroun, par avion cette fois-ci.

Limitée d'abord à des essais autour de Yaoundé et de Dschang, la pisciculture s'est étendue sur l'ensemble du Territoire à partir de 1951 . Fin 1951, on comptait 8 hectares d'étangs, 66 fin 1952, 154 fin 1953, 183 hectares représentant 4.778 étangs fin 1954 et 211 hectares représentant 5.364 étangs fin 1955.

D'abord conçue sur un plan analogue à celui du Congo Belge en 1948 (cf. Compte rendu de la conférence piscicole anglo-belge, Élisabethville 1948), la pisciculture a rapidement évolué au Cameroun vers la forme de l'étang ou bassin familial. Nous verrons plus loin les raisons de cette évolution. Les étangs se répartissaient ainsi' fin 1955 dans les quatre zones principales décrites plus haut :

\begin{tabular}{|c|c|c|c|}
\hline ZONES & $\begin{array}{l}\text { Nombre } \\
\text { d'étangs }\end{array}$ & $\begin{array}{c}\text { Superficie } \\
\text { couverte } \\
\text { en hectares }\end{array}$ & $\begin{array}{c}\text { Superficie } \\
\text { moyenne des } \\
\text { étangs en } \mathrm{m}^{2}\end{array}$ \\
\hline Plateaux du Centre.......... & 127 & 43,75 & 3.445 \\
\hline Plateaux de l'Est............... & 149 & 35,04 & 2.351 \\
\hline Montagnes de l'Ouest. & 4.992 & 102,17 & 205 \\
\hline Plateaux de l'Adamaoua........... & 88 & 4,07 & 462 \\
\hline
\end{tabular}




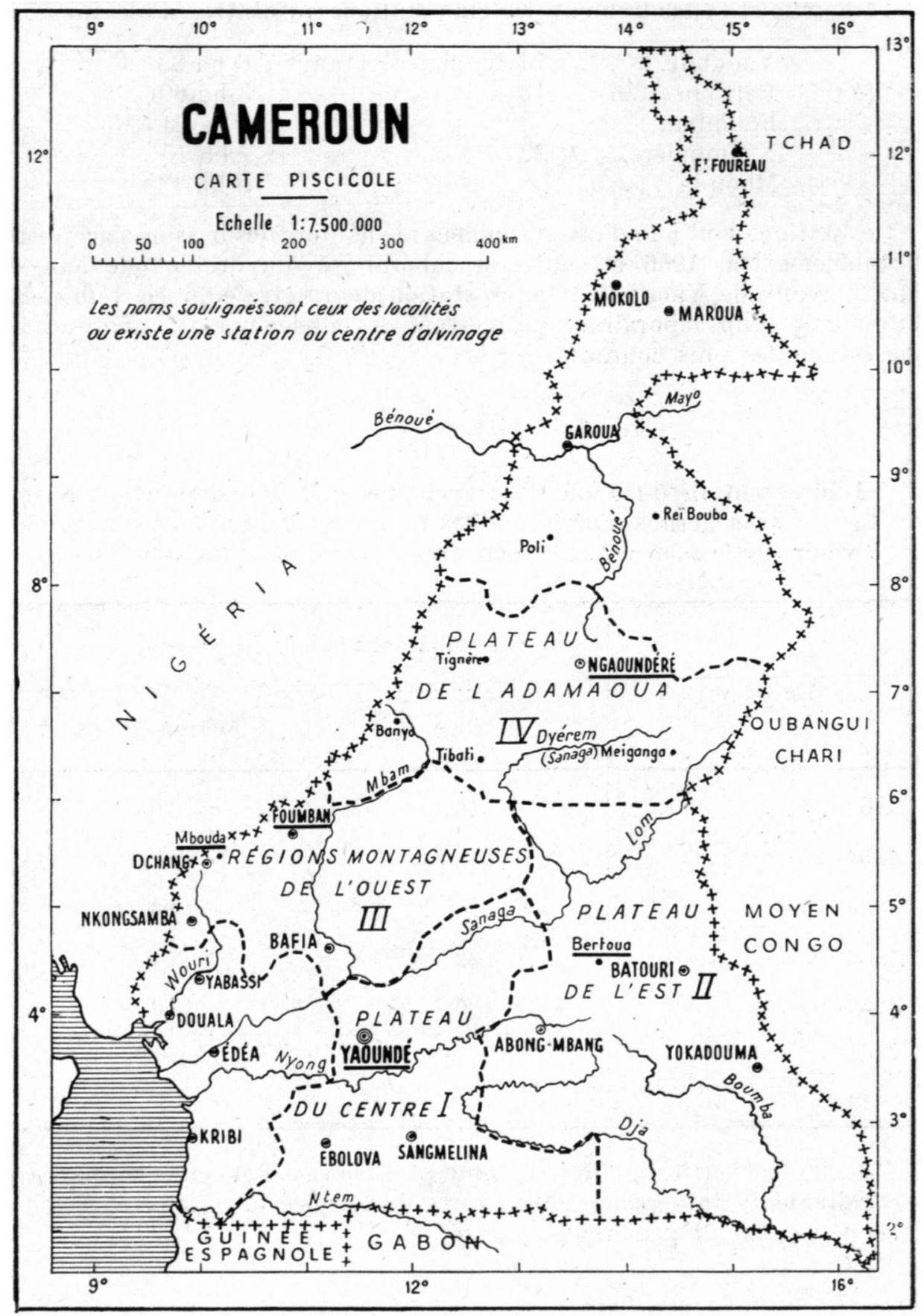


A cette répartition des étangs de pisciculture correspondent, pour la recherche et l'encadrement, les cinq stations ou centres d'alevinage :

$$
\begin{aligned}
& \text { Yaoundé. .... } 14 \text { bassins ou étangs : } 8 \text { ha } 85 \\
& \text { Bertoua...... } 13-\quad-\quad \text { : } 3 \text { ha } 69 \\
& \text { Foumban ..... } 8 \text { 8 — _ } \quad \ldots \text { : } 0 \text { ha } 21 \\
& \text { Ngaoundéré... } 29 \quad-\quad \ldots \quad \text { : } 2 \text { ha } 15 \\
& \text { Mbouda..... } 8-\quad-\text { : } 0 \text { ha } 43
\end{aligned}
$$

Ces stations sont loin d'être terminées : le nombre des bassins s'accroîtra notablement en 1956 et, outre le laboratoire d'hydrobiologie annexé aux bureaux de Yaoundé, chaque station devra être équipée d'un petit bureau et d'un laboratoire permettant les prélèvements, analyses et dissections les plus courants.

L'intervention du Plan de Développement Économique et Social du Cameroun a permis d'arriver à ces résultats. Les dotations accordées ou à venir sur le plan actuellement en exécution sont les suivantes :

\begin{tabular}{||c|c|c|}
\hline \multirow{2}{*}{ Exercices } & \multicolumn{2}{|c|}{ Montant : Millions de francs } \\
\cline { 2 - 3 } & CFA & Métropolitains \\
\hline $1953-1954 \ldots \ldots \ldots \ldots \ldots$ & 10 & 20 \\
$1954-1955 . \ldots \ldots \ldots \ldots \ldots$ & 16,25 & 32,5 \\
$1955-1956 \ldots \ldots \ldots \ldots \ldots$ & 18 \\
$1956-1957 \ldots \ldots \ldots \ldots \ldots$ & 32 & 64 \\
$1957-1958 . \ldots \ldots \ldots \ldots \ldots$ & 30 & 60 \\
\hline & 106,25 & 212,5 \\
\hline
\end{tabular}

Le budget territorial du Cameroun participe à cet effort en supportant les traitements du personnel des cadres du Service des Eaux et Forêts, mais non ceux du personnel contractuel.

L'organisation de la Pisciculture peut s'analyser brièvement ainsi : Recherche et Développement de la production sont étroitement associés, ce qui est normal dans une activité aussi récente. La direction de chacune 


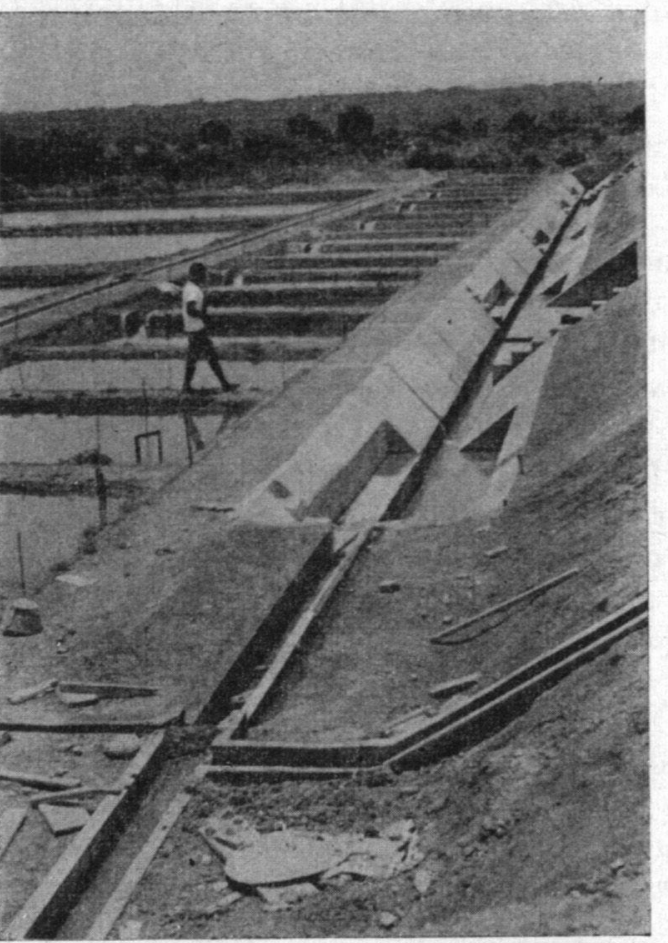

Centre d'alovinage de NGAOUNDERE (Adamaoua)

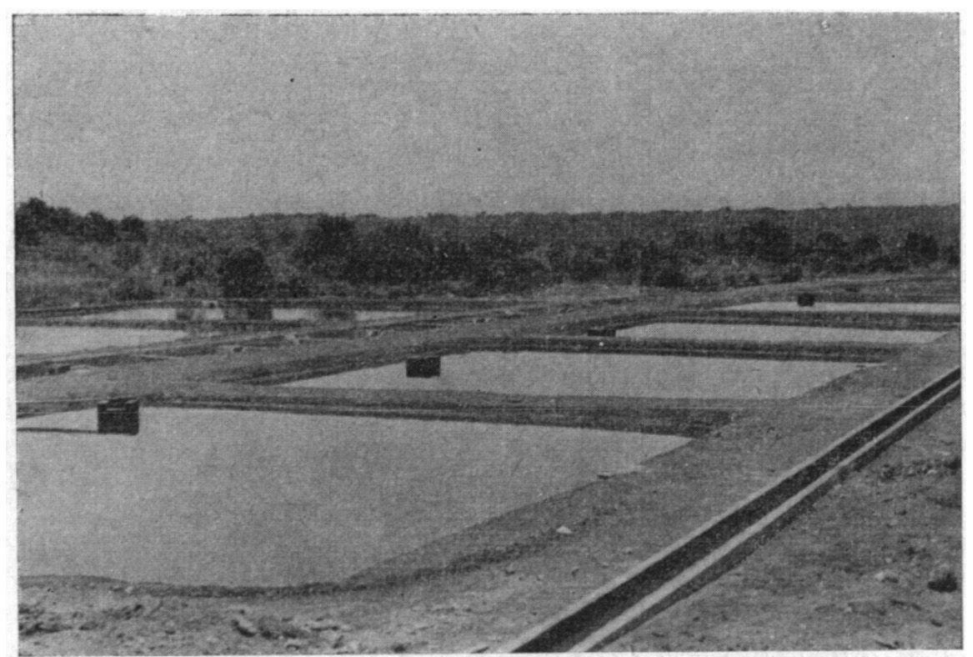

Contre d'alevinage de NGAOUNDERE (Adamaoua) 
des quatre stations ou centres d'alevinage est assurée par l'Officier des Eaux et Forêts de la circonscription forestière, assisté le plus souvent d'ingénieurs des travaux ou d'agents contractuels.

L'application des travaux des stations est dirigée vers des unités modèles de pisciculture, composées de quelques bassins du type adapté à la région, qui servent de relais pour les alevinages et, comme leur nom l'indique, de modèle aux pisciculteurs privés. L'encadrement de ceux-ci est assuré par des moniteurs itinérants dotés autant que possible de bicyclettes. Là où elle fonctionne suivant le schéma prévu, cette organisation donne d'excellents résultats; malheureusement, les difficultés de recrutement et de formation de personnel suffisamment qualifié tant africain qu'européen, en retardent actuellement la mise en place.

D'autre part, le personnel des cadres des Eaux et Forêts, même dans les régions où la pisciculture est la plus développée, est trop souvent sollicité par de nombreuses tâches forestières, mais extra-piscicoles.

Nous avons vu plus haut que, conçue à l'échelon industriel ou semiindustriel, la pisciculture est venue rapidement à l'échelon familial. Tous les premiers étangs construits au Territoire par des unités administratives, sociétés ou collectivités, étaient de grands étangs de barrage souvent d'une technique un peu rudimentaire, iaute de renseignements. Aussi les accidents n'ont-ils pas été rares. De plus, la gestion d'un grand étang est assez onéreuse pour qui veut bien s'en occuper et notamment donner la nourriture artificielle ; elle présente encore l'inconvénient de donner beaucoup de produits d'un seul coup, sans possibilités sérieuses de récoltes intermédiaires. Aussi, en 1952, l'apparition dans l'Ouest des premiers bassins familiaux a-t-elle été l'amorce d'une évolution qui se poursuit actuellement. D'une part, l'Africain a le sens collectif peu développé, au moins à l'intérieur des collectivités actuelles qui sont souvent très artificielles; d'autre part, la construction d'un bassin familial creusé est simple, sa gestion est peu coûteuse ; les propriétaires en font souvent plusieurs et peuvent ainsi échelonner leurs récoltes, les pêches intermédiaires sans vidange étant elles-mêmes beaucoup plus faciles que dans le cas d'un grand étang.

Malheureusement, il n'est pas possible partout de trouver facilement l'emplacement de bassins familiaux et, comme nous l'avons vu plus haut, les plateaux du Centre et de l'Est sont défavorisés à ce point de vue, en raison de la configuration de leur relief.

Le développement des bassins familiaux s'est donc fait d'une manière spectaculaire surtout dans l'Ouest. La facilité de réalisation de ces bassins a conduit à l'excès inverse de ce qui s'est produit dans le Centre et l'Est. Les autochtones se sont engoués de la pisciculture et ont réalisé des étangs dans un temps record, si vite que le personnel d'encadrement n'a pu suivre leur rythme. Les statistiques se sont gonflées au-delà de tout espoir, mais cette réussite incontestée pose maintenant de gros 


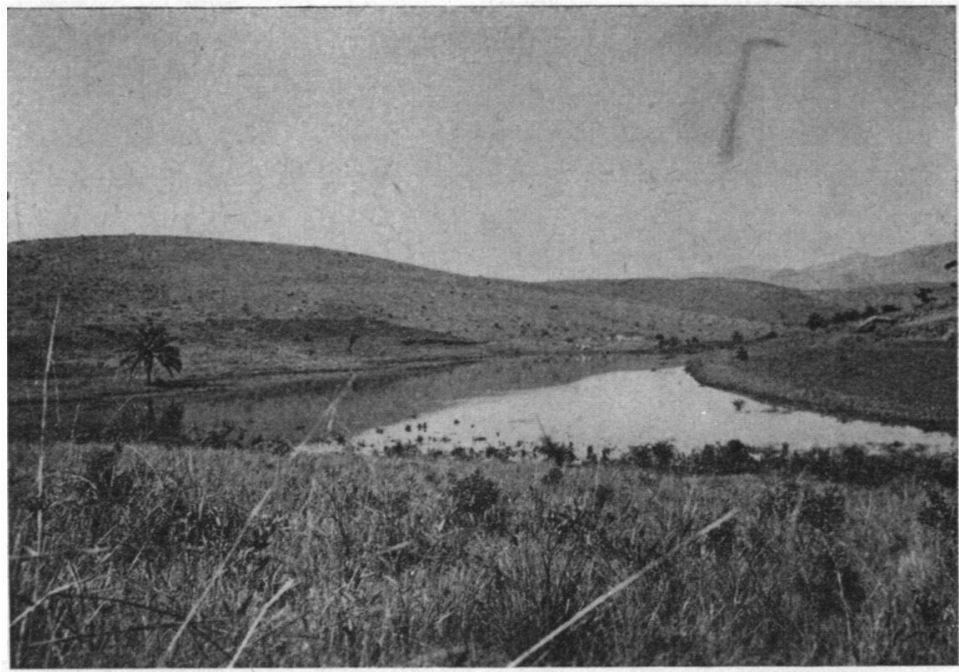

Etang de barrage a KOUPA-MATAPIT (Subdivision de FOUMBAN)

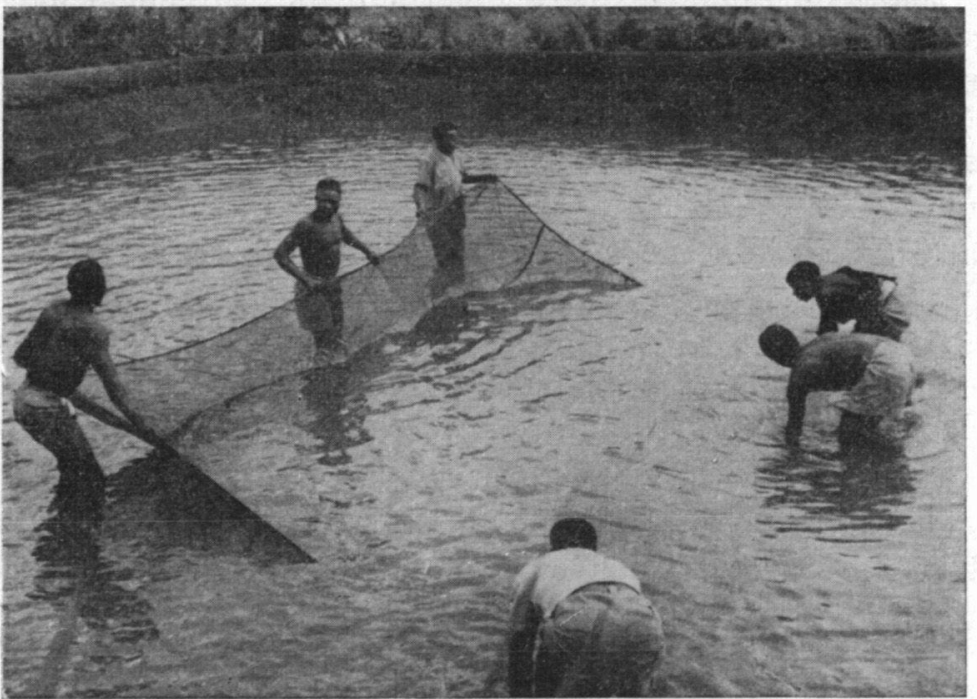

Poche dans un étang tamilial à FONGO-TONGO (Subdivision de DSCHANG) 
problèmes de correction d'aménagement et de gestion. Ces problèmes sont d'autant plus ardus que l'Africain s'emballe vite, mais se décourage non moins rapidement.

Dans tout ce qu'il a réalisé sur les conseils des Européens, l'Africain a vu des sources de profits pécuniaires; tel a été le cas de la banane et du café dans l'Ouest, du cacao dans le Centre. Tel n'est pas le cas du poisson d'étang qui, sauf cas exceptionnels, est le plus souvent un article de consommation familiale. Cette vérité s'impose difficilement; sa manifestation constitue une grande partie de la tâche des agents de la pisciculture : expliquer au pisciculteur africain qu'il ne doit pas compter sur la venue d'un courtier qui lui achètera sa récolte de poisson, mais qu'il doit utiliser celui-ci à la nourriture familiale.

Jusqu'ici les Tilapia melanopleura et surtout macrochir ont seuls été employés en pisciculture au Cameroun. Quelques autres espéces (Tilapia nilotica, Tilapia flavomarginata) sont en cours d'essai, mais quelle que soit l'espèce, tous les Tilapia, au moins sous les latitudes situées entre le $2^{\mathrm{e}}$ et le $8^{\mathrm{e}}$ parallèles Nord, semblent présenter le même nient : une reproduction trop abondante et trop précoce (1).

La taille de maturité sexuelle varie; elle est plus faible pour le Tilapia melanopleura que pour le Tilapia macrochir ou le Tilapia nilotica, mais pratiquement tous les étangs donnent le mème résultat : trop de poissons de petite taille, même si, par ailleurs, le rendement total en poids est intéressant. Or, l'Africain, même celui qui n'exploite qu'un étang familial, recherche toujours le gros poisson, fût-il mème de moindre qualité. De ceci découle la nécessité absolue d'augmenter la taille moyenne du poisson produit, faute de quoi la pisciculture risque de sombrer au Cameroun.

Quatre ordres de remèdes peuvent être envisagés :

- la nourriture artificielle, les amendements et les engrais;

- l'élevage des sexes séparés ;

- l'introduction de prédateurs;

- la substitution d'un autre genre au Tilapia.

La nourriture artificielle (feuilles, son de riz, tourteaux divers, déchets d'abattoir) a fait l'objet de nombreuses expériences, spécialement à la station de Yaoundé, sur les Tilapia melanopleura et macrochir. Dans l'ensemble, les résultats obtenus sont assez semblables à ceux de la station de la Djoumouna à Brazzaville. Mais la taille moyenne du poisson produit n'est guère améliorée. D'autre part, cette nourriture est chère au Cameroun et les comptes d'exploitation des étangs nourris

(1) Dans toute la zone à pisciculture du Cameroun et aux altitudes inférieures a 1.500 mètres, les Tilapia frayent toute l'année. Au-dessus de 1.500 mètres, il semble que la reproduction s'arrête en saison sèche, mais cette constatation n'intéresse que quelques rares villages de l'Ouest. 
artificiellement dans les stations ne font apparaître que des bénéfices faibles ou nuls. Enfin, l'indigène, dans son exploitation familiale, ne nourrit pas plus son poisson qu'il ne nourrit ses chèvres, moutons, cochons ou poulets. On ne peut guère compter que sur la " nourriture automatique" constituée par les déchets issus des travaux ménagers exécutés au bord de l'étang; encore faut-il pour cela que l'étang ne soit pas trop loin des habitations, ce qui est assez rare. Aussi, dans les conditions actuelles au Cameroun, tous les calculs effectués sur les bilans nutritifs des étangs n'ont-ils qu'un intérêt purement théorique.

L'amendement calcique, dans des eaux à degré hydrotimétrique très faible, pourrait sans doute donner des résultats intéressants. Malheureusement, on ne peut se procurer sur place ni marne, ni chaux. Il faudrait avoir recours à la chaux d'importation, mais son prix, 20 à 30 francs CFA (40 à $60 \mathrm{fr}$. français) le kilogramme, est prohibitif. On peut faire le même reproche aux engrais chimiques dont la manipulation est, de surcroit, délicate. L'engrais organique, lui, est introuvable pour le moment dans un pays où l'élevage en stabulation est inconnu. L'introduction de cette dernière pratique pour les moutons et les porcs est inscrite au programme d'économie rurale du Territoire. Il sera intéressant de coupler, à l'instar de ce qui s'est fait en Indonésie, étangs avec porcheries ou bergeries, les premiers recevant les eaux d'écoulement des seconds (1).

L'élevage des sexes séparés semble théoriquement intéressant, mais une telle pratique sera cantonnée obligatoirement dans les stations d'essais. On ne peut espérer, en effet, l'introduire dans la pisciculture familiale, le sexe des Tilapia étant trop difficile à reconnaître dans le jeune âge.

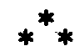 \\ Introduction de prédateurs.}

Les essais dans ce domaine sont à peine amorcés au Cameroun, mais les résultats déjà obtenus, tant en Indonésie qu'au Congo Belge, semblent indiquer que cette voie est intéressante.

Il était logique de songer d'abord aux Clarias sp. que l'on trouve presque toujours dans les étangs à Tilapia. Malheureusement, tous les essais tentés se sont révélés jusqu'à présent négatifs; les Clarias, tout en se reproduisant eux-mêmes parfaitement bien dans les étangs à Tilapia, sont impuissants à freiner la pullulation des alevins de ces derniers. La même constatation a d'ailleurs été faite en Indonésie.

En outre, les Clarias sont difficiles à contrôler car ils disparaissent facilement dans la boue.

(1) Des essais sont actuellement en cours à la station de Yaoundé pour faire alterner pisciculture et riziculture sur l'assiette d'un même étang: il est possible que la vase riche en phosphore du fond des étangs profite au riz et qu'inversement la culture du riz profite à l'élevage subséquent de Tilapia. 
Lors de la vidange récente d'un marécage qualifié étang à Tilapia dans l'Est du Territoire, on a observé une assez grande quantité, non mesurée malheureusement, d'Hepsetus odoe qui avaient fait disparaître, semble-t-il, les petits Tilapia. Ce Characinidé prédateur va faire l'objet d'essais systématiques.

L'Hemichromis fasciatus, d'après de récents travaux belges, semble le prédateur le plus intéressant actuellement malgré sa faible taille. Des essais de ce Cichlidé en mélange avec des Tilapia sont en cours.

Un récent travail indonésien fait allusion à des résultats intéressants pour la sélection des Tilapia obtenus avec l'Ophiocephalus (probablement striatus). Il y aurait intérêt à essayer l'espèce voisine Parophiocephalus obscurus, assez fréquente au Cameroun.

La liste des prédateurs à essayer n'est pas close : les Polyptères, les Schilbe, dont la pisciculture a été tentée autrefois au Congo Belge, les Eutropius et, peut-être même, le Lates, pourront également être utilisés (1).

Une nouvelle pisciculture est en cours d'essai au Cameroun : celle de l'Heterotis niloticus. Cette espèce microphage a, en eau libre, une croissance extrêmement rapide; il reste à déterminer si, en eau close, la reproduction excessive ne freinera pas la croissance comme chez le Tilapia. Un individu, isolé au milieu de Tilapia dans un bassin à Yaoundé, est passé, en 4 mois $1 / 2$, de 4 à 21 centimètres de longueur totale.

Jusqu'à présent, enfin, la faune locale ne nous a pas fourni de Cyprinides dont la pisciculture semble présenter de l'intérêt.

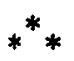

Le nombre des étangs de pisciculture s'accroît actuellement à un rythme extrêmement satisfaisant, preuve que cette activité rencontre toujours la faveur de l'autochtone, sauf en quelques points où les Africains ont été abusés par une propagande exagérée. Le Tilapia n'est pas une mine d'or, c'est entendu, mais il constitue un sérieux appoint à la nourriture familiale et les autochtones s'en rendent compte. La meilleure preuve en est que les progrès les plus spectaculaires de la pisciculture se font en ce moment en région Bamiléké, pays à population dense de montagnards qui entendent fort bien leurs intérêts. Il appartient maintenant au service des Eaux et Forêts du Cameroun de pousser ses recherches pour accroître le rendement en valeur des étangs.

(1) On n'a pas mentionné un essai d'introduction de Black-bass actuellement en cours dans l'Ouest, à $\mathbf{1 . 4 0 0}$ mètres d'altitude, en mélange avec des Tilapia. 